He showed very clearly, using a rather fearsome phosphate-specific reagent, that the lac repressor only binds on one side of the double helix. Second, both E. De Robertis (MRC, Cambridge) and D. Brown described experiments done in J. Gurdon's laboratory at Cambridge, which involved the injection of either frog 5S DNA or Drosophila histone DNA into the nuclei of frog oocytes (a seemingly impossible manipulation). Both experiments suggested that the foreign DNAs were transcribed specifically to generate in the first case normal 5S RNA and in the second histone proteins, detected by two-dimensional fractionation techniques. These exciting results suggest that frog oocytes may well provide an invaluable assay system for purified eukaryotic gene expression.

\section{Nova Sagittae 1977 and Vulpeculae 1976}

\section{from David $W$. Hughes}

Tenacity and a truly astronomical memory are two attributes specifically required by the amateur astronomers who successfully search the sky for novae. Tenacity, because discoveries are few and far between-about 300 hours of painstaking observation being required to discover a nova. The encyclopaedic memory is also essential, the relative positions and brightness of about 10,000 stars must be known so that the interloper, the star which has suddenly and dramatically increased in brightness, can be easily recognised.

The last two novae found have been discovered by British amateur astronomers, the most recent one by John Hosty, a Huddersfield postman, on 7 January, 1977 in the constellation of Sagitta (the actual position is right ascension $19^{\mathrm{h}} 37^{\mathrm{m}} 08.18^{\mathrm{s}}$, declination $+18^{\circ} 00^{\prime} 58.2^{\prime \prime}$, equinox 1950.0). This has been reported in Circulars $3025 / 7$ and 3030 of the International Astronomical Union's Central Bureau for Astronomical Telegrams. It was discovered at a magnitude of 7.2 (about three times less bright than stars visible to the naked eye, which have magnitudes less than 6.0) and has been getting steadily fainter since its discovery, as shown in Fig. 1. Most novae will decrease in brighitness by about 3 magnitudes from their maximum value in about $40 \mathrm{~d}$ and then continue getting fainter at a slightly diminished rate.

The previous nova discovery was by George Alcock, a retired school master from Peterborough, his nova being in Vulpecula (right ascension $19^{\mathrm{h}} 27^{\mathrm{m}} .04^{\mathrm{s}}$, declination $+20^{\circ} 21^{\prime} 43^{\prime \prime}$ ) and was near its maximum brightness at magnitude 6.5 at discovery (IAU Circular No. 2997). As seen in Fig. 1 the nova became 3 magnitudes fainter than maximum in $42 \mathrm{~d}$ and 6 magnitudes fainter in about $68 \mathrm{~d}$. Interestingly both novae are in the Milky Way and are very close to each other in the sky.

The instruments used to detect novae are relatively simple in comparison to other astronomical apparatus. Both Hosty and Alcock used binoculars, in fact Hosty used only half a pair of binoculars as the other half had been unfortunately broken at some time. The two instruments were $10 \times 50$ and $15 \times 80$ respectively, the first figure being the magnification and the second figure the objective lens diameter in millimetres. They would have fields of view of about $6^{\circ}$ and $4^{\circ}$ in diameter, the Hosty instrument detecting stars down to about 10th magnitude under excellent sky and seeing conditions. The field of view would contain, on average, 30 stars of 8 th magnitude and just over 200 brighter than 10th. The daunting task of the nova hunter is to pick out the new star as he sweeps slowly over the 10,000 or so stars brighter than 8th magnitude that can be seen from a good observing sight at any one time.

What is the use of discovering novae? Well, apart from the sudden rise to fame for the finder, these stars are of considerable intrinsic interest, being at a fascinating stage in their evolutionary history. Novae occur just before a star finally dwindles to become a white dwarf. All the nuclear energyproducing reactions converting hydrogen and helium into higher atomic number elements have ended, and the star is getting its energy simply from the gravitational potential energy that is slowly released as it shrinks. The prenova star is typically of spectral type $O$ or B, corresponding to a surface temperature of 20,000 to $40,000 \mathrm{~K}$, with a radius about 0.3 solar radii and a mass between 0.2 and 2.5 solar masses. The absolute magnitude is around +4.5 . On explosion the magnitude jumps by 11 , equivalent to an increase in brightness of about 25,000 times. During the outburst the star loses on average 0.001 solar masses, the energy emitted in the few days of outburst being about $10^{45}$ ergs, corresponding to the energy emitted by the Sun over 2,000 yr. From spectral observations it can be seen that the luminous layers of the star are expanding outwards at velocities around 1,000 to $2,500 \mathrm{~km} \mathrm{~s}^{-1}$ in a rather non-uniform fashion, causing the superposition of absorption and emission features in the line spectra. At maximum the nova reaches an absolute magnitude of about -8.0 the expanding gas cloud having a radii of about 100 solar radii. After maximum the nova slowly fades into insignificance, back to the same magnitude as the pre-nova star. By care-

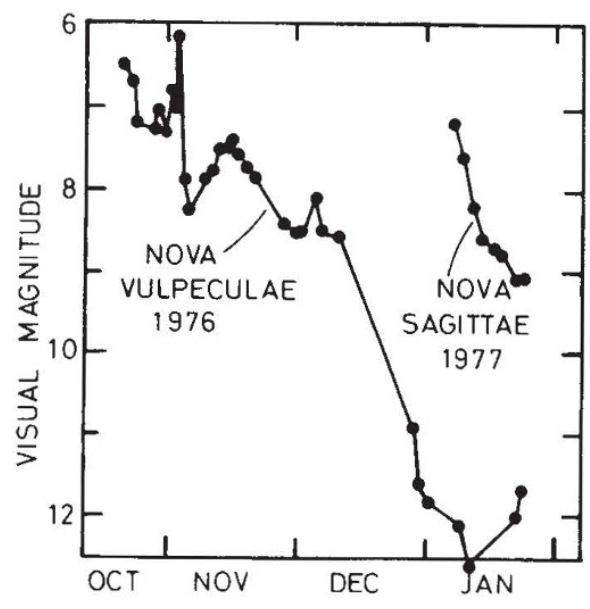

Fig. 1 The visual magnitude of the novae as a function of time. A change in magnitude by one unit is produced by a change in brightness by a factor of 2.51

fully searching the Palomar Sky Survey photographic plates (IAU Circular 2998) the pre-nova star of nova Vulpeculae has been found and is a blue star of photographic magnitude 18.3 . A search for the pre-nova of Sagittae 1977 has revealed three stars at the plate limit of ahout 20th photographic magnitude within $\sim I^{\prime \prime}$ of the nova's position.

In the Galaxy there may be about 50 novae a year, only a few $\left(2.2 \mathrm{yr}^{-1}\right.$ on average) being discovered, the rest being hidden behind dense interstellar dust clouds or being too faint at maximum.

The general excitement caused when one is discovered and the wealth of knowledge gained about this fascinating stage of stellar evolution is ample justification for the arduous and tireless search that worthy amateur astronomers like Hosty and Alcock conduct. It is very unfortunate however that so little is known about the pre-nova state of the star. So far only one spectrum, that of V603 Aquilae, has been recorded before the star became a nova. Our knowledge of what causes a star to suddenly explode in this unexpected way is thus pitifully small.

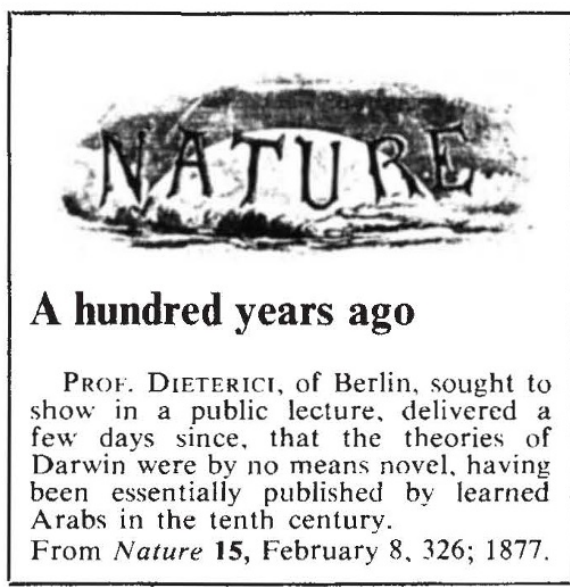

